\title{
Efectos del tratamiento térmico y del método de colección sobre la estructura cristalina de las nanofibras electrohiladas de ácido poli (láctico)
}

\author{
Effects of heat treatment and the method of collection on the crystal structure of the \\ poly lactic acid electrospinned nanofibers
}

\author{
Edwin Gómez-Pachón $^{1 *} \quad$ Ricardo Vera-Graziano ${ }^{2} \quad$ Raúl Montiel $^{3}$ \\ William Cardenas-Aguazaco ${ }^{4} \quad$ Andrés Felipe Ochica $^{4}$ \\ Recibido 29 de octubre de 2017, aceptado 13 de septiembre de 2018 \\ Received: October 29, 2017 Accepted: September 13, 2018
}

\begin{abstract}
RESUMEN
Estudios anteriores indican que las nanofibras de ácido poli (láctico) (PLA) obtenidas por electrohilado reportan una baja resistencia mecánica. Por ello se pretende mostrar los cambios en las propiedades mecánicas de módulo de Young y resistencia a la tensión de las nanofibras de PLA por efectos del tipo de colección y de tratamiento térmico. Para ello se prepararon nanofibras de PLA por electrohilado colectadas aleatoria y ordenadamente con la aplicación de un tratamiento térmico a $80^{\circ} \mathrm{C}$ por 8 horas con el fin de incrementar el entrecruzamiento y la fase cristalina del andamio. Las nanofibras fueron caracterizadas por medio de microscopía electrónica de barrido (SEM), microscopía electrónica de transmisión (TEM), dispersión de rayos X a bajo ángulo (SAXS), calorimetría diferencial de barrido (DSC) y una evaluación a la resistencia a la tensión. Los resultados mostraron nanofibras orientadas con una estructura semicristalina claramente definida, con un menor diámetro promedio de fibra y un notorio aumento de resistencia a la tensión como del Módulo de Young en relación a las depositadas aleatoriamente. Por otra parte, el tratamiento térmico influenció en el diámetro promedio y la porosidad final del andamio, pero no generó un cambio notorio en las propiedades mecánicas en estudio. Por lo tanto, se concluye que el proceso de colección rotatorio genera un mayor ordenamiento molecular, incremento en las propiedades mecánicas en estudio, mientras el recocido extiende la cristalinidad de las nanofibras pero no afecta estas propiedades.
\end{abstract}

Palabras clave: Acido Poli (láctico), electrohilado, nanofibras, cristalinidad, propiedades mecánicas.

\section{ABSTRACT}

Previous studies indicate that poly (lactic acid) (PLA) nanofibers obtained by electrospinning report a low mechanical strength. Therefore, it is intended to show the changes in the mechanical properties of Young's modulus and tensile strength of PLA nanofibers due to the type of collection and thermal treatment. For this, PLA nanofibers were prepared by electrospinning collected randomly, and orderly with thermal treatment was carried out at $80^{\circ} \mathrm{C}$ for 8 hours to increase the cross-linking and the crystalline phase of the scaffolding. The nanofibers were characterized by means of scanning electron microscopy

1 Grupo de investigación Diseño, Innovación y Asistencia Técnica de Materiales Avanzados DITMAV. Facultad Duitama. Universidad Pedagógica y Tecnológica de Colombia (UPTC), Carrera 18 con Calle 22, Duitama, Colombia. E-mail: edwin.gomez02@uptc.edu.co

2 Instituto de Investigaciones en Materiales, UNAM Apdo. postal 70-360 Cd. Universitaria, CDMX, México.

E-mail: graziano@unam.mx

3 Departamento de polímeros. Universidad Autónoma Metropolitana-Iztapalapa (UAM-I), CDMX, México. E-mail: luar@xanum.uam.mx

4 DANUM, Facultad de Ciencias Básicas. Universidad Pedagógica y Tecnológica de Colombia (UPTC). Avenida Central del Norte 39-115, Tunja, Colombia. E-mail: williamantonio.cardenas@uptc.edu.co; andres-felipe21@ hotmail.com

* Autor de correspondencia: edwin.gomez02@uptc.edu.co 
(SEM), transmission electron microscopy (TEM), low angle X-ray scattering (SAXS), differential scanning calorimetry (DSC), and stress evaluation. The results showed oriented nanofibers with a clearly defined semicrystalline structure and a smaller average fiber diameter and a notorious increase of tensile strength concerning those collected randomly. Additionally, the thermal treatment influenced the average diameter and the porosity of the scaffolding but did not generate a significant change in the mechanical properties. Therefore, it is concluded that the rotary collection process generates a higher molecular order, increase in the mechanical properties under study, while annealing extends the crystallinity of the nanofibers but does not affect these properties.

Key words: Poly Lactic Acid, electrospinning, nanofibers, crystallinity, mechanic properties.

\section{INTRODUCCIÓN}

La medicina regenerativa, es un campo interdisciplinario que combina los principios e innovaciones de la ingeniería y ciencias de la vida con el propósito de la mejora, reparación o sustitución de la función de un tejido u órgano [1]. En este campo de la ciencia se combinan células, andamios (matriz extracelular artificial) y tecnologías de diseño y fabricación de los nuevos tejidos u órganos, dado que cada tejido u órgano en nuestro cuerpo está compuesto de células del parénquima (células funcionales) y células madre mesenquimales (células de apoyo) contenidos dentro de una matriz extracelular (MEC) para formar un microambiente, los cuales en conjunto forman tejidos y órganos [2].

Entre las propiedades más importantes que deben poseer los andamios fabricados por electrohilado está la capacidad de promover la adhesión, crecimiento y diferenciación de células in-vitro, además de su biocompatibilidad, biodegradabilidad y soporte celular [2]. Para el desarrollo de andamios se han utilizado diferentes materiales tales como: sintéticos orgánicos (polímeros), sintéticos inorgánicos (hidroxiapatita, fosfato tricálcico, yeso, cerámica de vidrio), orgánicos de origen natural (colágeno, ácido hialurónico) e inorgánicos (hidroxiapatita) [3-7].

Los andamios celulares a base de fibras con diámetros nanométricos tienen varias ventajas en comparación con otro tipo de andamios. Por ejemplo, las nanofibras poseen mayor probabilidad de que se adhieran las células, así como una mejor porosidad para la entrada y salida de nutrientes, control de la degradación, entre otras. Sin embargo, dada su baja densidad y estructura requieren de propiedades mecánicas que permitan soportar todo el proceso que implica el desarrollo de los tejidos [8]. Las nanofibras poliméricas pueden presentar propiedades físicas, químicas y en algunos casos propiedades mecánicas similares a las que poseen algunos tejidos vivos (hueso, cartílago, músculo, venas y arterias). Además presentan propiedades como: elevada relación superficie/volumen, buena resistencia mecánica, posibilidad de funcionalizar su superficie y facilidad para adoptar la forma de un tejido, cuando se utiliza como andamio celular [1]. Las nanofibras poliméricas garantizan el soporte de las células durante un período de tiempo, para luego degradarse a medida que se obtiene el nuevo tejido, así como una respuesta biológica favorable del organismo receptor [9].

La técnica de electrohilado ha mostrado varias ventajas para fabricar redes o andamios de microfibras y nanofibras, ya que permite controlar el diámetro de las fibras, así como la estructura del andamio (dirección de nanofibras, porosidad, estructura, entre otros), lo que facilita la manufactura controlada de andamios celulares útiles en la ingeniería de tejidos $[9,10]$. Los andamios poliméricos producidos por el proceso del electrohilado resultan ser una alternativa a los inconvenientes que presentan otros tipos de materiales porosos, que no permiten el desplazamiento de fluidos dentro de sus poros. El proceso de electrohilado ofrece la posibilidad de mejorar algunas propiedades de los polímeros para satisfacer los requerimientos mecánicos del andamio celular y del ambiente biológico al que va a estar sometido. Otra ventaja que presentan cuando el andamio se manufactura por electrohilado es su versatilidad para construir formas complejas en $2 \mathrm{D}$ y $3 \mathrm{D}[9,11]$.

En estudios realizados con anterioridad se desarrolló un andamio con PLA e hidroxiapatita 
para la regeneración dental, utilizando la técnica de electrohilado [12]. Se obtuvieron andamios de PLA mezclado e injertado con nano partículas de hidroxiapatita. Se lograron buenas propiedades mecánicas, químicas y biológicas, en comparación con otros andamios desarrollados hasta ese momento. Sin embargo, no se estableció una relación entre la caracterización estructural y sus propiedades mecánicas. Varios estudios se han enfocado en el análisis de las propiedades elásticas y resistencia a la tensión de nanofibras poliméricas $[13,14]$ y a la observación de la estructura superficial de nano y microfibras por técnicas como microscopía electrónica de barrido (SEM) [9], los cuales brindan algunas descripciones de la morfología, pero no han logrado hacer una correlación de la morfología interna de las nanofibras con sus propiedades elásticas, para usarse en el diseño de andamios celulares y materiales compuestos a base de nanofibras poliméricas.

El estudio reportado por You et al. [15], indica una disminución en el área de las fibras de PLA al ser sometidas a una temperatura de $180^{\circ} \mathrm{C}$, pero no se estudió el efecto de los diferentes tipos de colector y del tratamiento térmico de recocido sobre las estructura semicristalina interna de las fibras de PLA.

El objetivo del estudio consiste en evaluar el efecto generado en las nanofibras de acuerdo al método de colección y del tratamiento térmico. Para ello se utilizan diferentes técnicas analíticas para determinar los cambios morfológicos de las nanofibras de PLA, analizando específicamente los efectos sobre las propiedades estructurales cristalinas. Lo anterior pretende correlacionar estas propiedades morfológicas con las propiedades mecánicas, las cuales dan un avance significativo al desarrollo de andamios para la utilización en el campo de la ingeniería de tejidos.

\section{MATERIALES Y MÉTODOS}

\section{Materiales}

El PLA fue adquirido de Ingeo, Cargill Dow Co (división Natureworks 2002D) en forma de pellets. Para la obtención de los andamios de PLA fue utilizado el solvente 2,2,2-Trifluoroetanol $\left(\mathrm{N}^{\circ} \mathrm{CAS}\right.$ 75-89-8) el cual fue adquirido de Sigma-Aldrich a una concentración de $99 \%$.

\section{Electrohilado}

Las soluciones de PLA fueron preparadas según la metodología usada por Vera et al. [12] usando 2,2,2-Trifluoroethanol (TFE) como solvente. Las soluciones fueron agitadas magnéticamente hasta lograr una solución homogénea. Los andamios se fabricaron utilizando el equipo de electrohilado (Figura 1) en el Instituto de Investigación en Materiales de la Universidad Nacional Autónoma de México (IIM-UNAM).

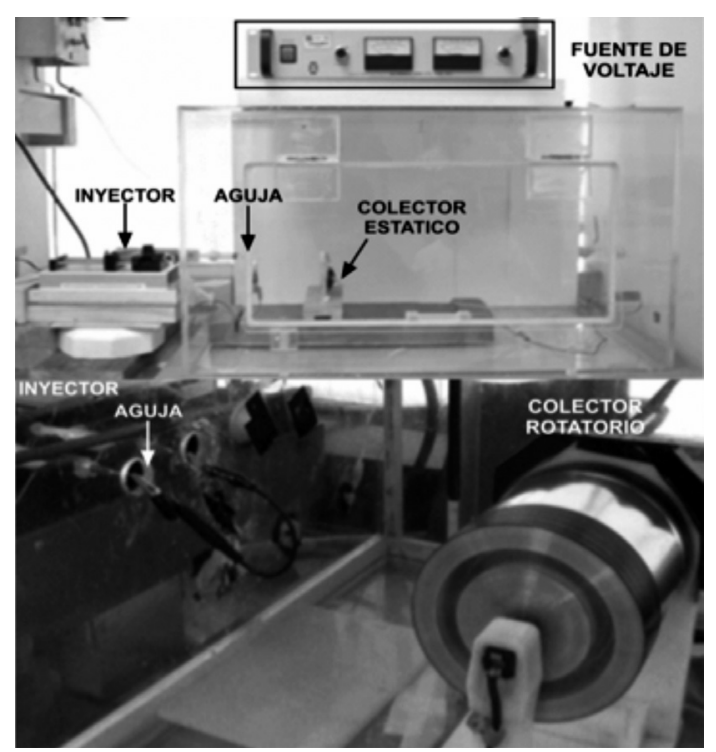

Figura 1. Equipo de electrohilado del IIM-UNAM.

Los parámetros utilizados en el electrohilado de las fibras colectadas aleatoriamente son descritos en la Tabla 1:

Tabla 1. Parámetros de electrohilado de las fibras colectadas aleatoriamente.

\begin{tabular}{|l|c|}
\hline \multicolumn{1}{|c|}{ Parámetro } & Valor \\
\hline Distancia del colector al inyector & $30 \mathrm{~cm}$ \\
\hline Voltaje & $15 \mathrm{KV}$ \\
\hline Velocidad de Inyección & $0,6 \mathrm{ml} / \mathrm{h}$ \\
\hline Temperatura del proceso & $25^{\circ} \mathrm{C}$ \\
\hline
\end{tabular}

Para la obtención de las nanofibras de PLA ordenadas, se modificó el voltaje y la velocidad del colector rotatorio. Los valores son descritos en la Tabla 2. 
Tabla 2. Parámetros de electrohilado usados en las fibras colectadas ordenadamente.

\begin{tabular}{|c|c|c|}
\hline Voltaje (KV) & RPM & $\mathbf{V}_{\tan }(\mathrm{m} / \mathrm{min})$ \\
\hline 13,6 & 3491 & 1096.7 \\
\hline 15 & 3876.9 & 1217.9 \\
\hline 18 & 4703.6 & 1477.7 \\
\hline
\end{tabular}

\section{Tratamiento post electrohilado}

Con el fin de mejorar las propiedades estructurales y mecánicas de las fibras, estas fueron sometidas a un proceso de calentamiento a $80^{\circ} \mathrm{C}$ durante 10 horas. La temperatura de este proceso fue dada de acuerdo a la temperatura de transición vítrea del polímero $\left(\mathrm{Tg}=60^{\circ} \mathrm{C}\right)$, con lo cual se asegura un cambio en la estructura interna del polímero. Las muestras con post tratamiento térmico se presentan en los resultados como NAR (Nanofibras colectadas aleatoriamente recocidas) y NOR (Nanofibras colectadas ordenadamente recocidas).

\section{Caracterización por SEM}

Con la ayuda de la microscopia electrónica de barrido (SEM) fue posible observar los aspectos morfológicos, diámetro y porosidad de las nanofibras de PLA. Para tal fin fue utilizado el equipo JEOLJSM-7600F (Japón) con filamento de Tungsteno, el cual poseen un cañón de electrones con un diferencial de 20.0 kV Para la realización de esta caracterización se tomó $0,5 \mathrm{~cm}^{2}$ de las nanofibras obtenidas de los diferentes tratamientos descritos anteriormente. Para el análisis fue necesario recubrir con oro a un vacío de 10E-8 Torr con una corriente de $30 \mathrm{~mA}$ durante 5 minutos. Se tomaron dos fotografías de cada probeta en diferentes lugares con aumentos entre 1000x y 50000x. Con las imágenes obtenidas se realizó un análisis del diámetro y porosidad de las nanofibras mediante software Image J.

\section{Caracterización por TEM}

Con la Microscopía Electrónica de Transmisión (TEM) es posible observar los cambios de estructura interna de las nanofibras. Las nanofibras fueron encapsuladas en una resina epóxica (Epon ${ }^{\circledR} 812$ ) y calentadas por tres días en un horno a $50{ }^{\circ} \mathrm{C}$. Posterior a este procedimiento, las nanofibras fueron cortadas en discos de $100 \mathrm{~nm}$ de espesor con un ultra micrótomo MRC-7000. Para obtener un buen contraste en las observaciones de TEM, las muestras fueron colocadas en un horno en presencia de tetróxido de rutenio durante 20 minutos, con el objetivo de oxidar el polímero y así poder observar claramente los contrastes entre las fases amorfas y las semicristalinas. El equipo utilizado en esta caracterización fue JEOL-2100f.

\section{Caracterización por DRX y SAXS}

La determinación de la estructura cristalina interna de las nanofibras fue llevada a cabo mediante caracterización por Dispersión de rayos X a bajo ángulo (SAXS), se empleó el equipo Xeuss SAXS/ WAXS (Francia), el cual posee un tubo de rayos $X$ Genix de baja divergencia y un detector Dectris pilatus $300 \mathrm{~K}$, con tubo de dispersión al vacío. Se usó el tubo de rayos $\mathrm{X}$ con un voltaje de $50 \mathrm{kV}$ y una corriente de 0,6 mA. La emisión de rayos $\mathrm{X}$ fue de 120 segundos de exposición y la dispersión se realizó a ángulos inferiores de 1 .

Este análisis se llevó a cabo al PLA en pellets, a las nanofibras colectadas aleatoriamente (NA) y a las nanofibras orientadas con una velocidad de $1480 \mathrm{~m} /$ $\min$ (NO $1480 \mathrm{~m} / \mathrm{min}$ ). La muestra de PLA y las nanofibras fueron analizadas con un espesor de la muestra de $0.5 \mathrm{~mm}$, mientras que el tamaño de las muestras se ajustaron al diámetro por donde pasa el haz de rayos $\mathrm{X}$.

\section{Análisis por Calorimetría diferencia de barrido (DSC)}

Las transiciones térmicas que presentan las nanofibras se determinaron por el equipo DSC 2910 TA Instruments. Las muestras se calentaron desde $0{ }^{\circ} \mathrm{C}$ hasta $200^{\circ} \mathrm{C}$ a una rampa de calentamiento de $10^{\circ} \mathrm{C} / \mathrm{min}$. Los termogramas obtenidos se analizaron por medio del software "TA Instruments Universal Analysis 2000".

\section{Caracterización mecánica de los andamios de nanofibras de PLA}

Se construyeron probetas ajustadas a la norma técnica ASTM D638, dado que actualmente no existe una norma específica para membranas de nanofibras. Se escogió esta norma porque está recomendada para membranas de materiales poliméricos con fibras reforzadas. Se usó la celda de carga de 50 gramos de fuerza de la empresa Honeywell Sensing \& Control, Model 31 (Miniature Load Cell). Para el ensayo se utilizó el equipo universal de ensayo marca MTS minibionix 858. La velocidad del ensayo fue de $10 \mathrm{~mm} / \mathrm{min}$. Los datos fueron procesados en 
MatLab 2010®. Se probaron 5 probetas por cada tratamiento.

\section{RESULTADOS Y DISCUSIÓN}

\section{Morfología por (SEM)}

En un estudio realizado por Gómez et al. [16] se comparó el método de colección de las nanofibras de PLA, usando un colector rotacional y un colector fijo. En ese estudio se utilizó una velocidad rotacional de 1100 y $1217 \mathrm{~m} / \mathrm{min}$ con el fin de determinar posibles cambios morfológicos y estructurales en las nanofibras. Se observó una relación en el diámetro de las nanofibras y la velocidad del colector, siendo a mayor velocidad mayor diámetro de las nanofibras. En el presente estudio se muestran los diámetros y la morfología de nanofibras de PLA obtenidas a una velocidad de $1480 \mathrm{~m} / \mathrm{min}$, además de los resultados obtenidos luego de realizar el proceso de calentamiento a las nanofibras. En la Figura 2 se puede apreciar las fibras obtenidas, encontrando una gran similitud respecto a la morfología de las fibras colectadas tanto aleatoria como ordenadamente. Las fibras no presentan microesferas (beads), fracturas o grietas, las cuales tienden a afectar las propiedades mecánicas de los andamios.
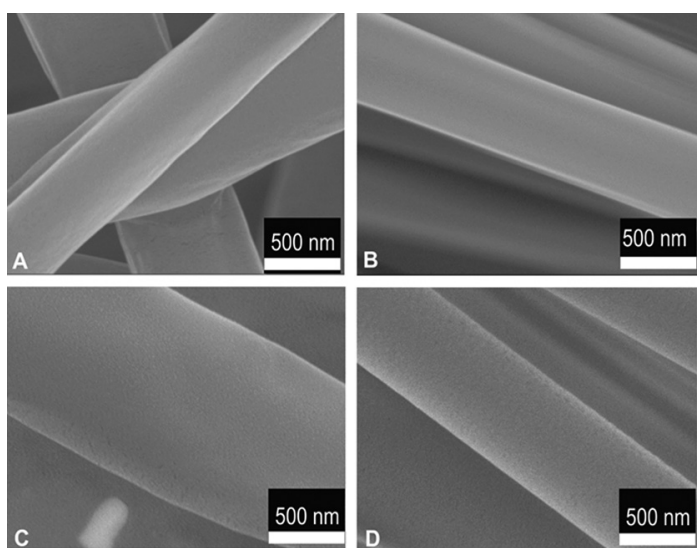

Figura 2. Micrografía electrónica de barrido de nanofibras: A. Colectadas aleatoriamente, B. Colectadas a $1100 \mathrm{~m} / \mathrm{min}, \mathrm{C}$. Colectadas a $1217 \mathrm{~m} / \mathrm{min}$ y D. Colectadas a $1480 \mathrm{~m} / \mathrm{min}$.

En la Tabla 3 se presentan los diámetros calculados de las nanofibras colectadas aleatoria y ordenadamente, así como de los diámetros de las nanofibras luego del proceso de recocido.
Tabla 3. Resultados de diámetro y porosidad en las nanofibras por microscopia electrónica de barrido.

\begin{tabular}{|l|c|c|}
\hline \multicolumn{1}{|c|}{$\begin{array}{c}\text { Tipo } \\
\text { de andamio }\end{array}$} & $\begin{array}{c}\text { Diámetro promedio } \\
(\mathbf{n m})\end{array}$ & $\begin{array}{c}\text { Porosidad } \\
\text { promedio } \\
(\%)\end{array}$ \\
\hline $\mathrm{NA}^{1}$ & $992+/-25,7$ & 37,6 \\
\hline $\mathrm{NAR}^{2}$ & $986+/-21,1$ & 44,3 \\
\hline $\mathrm{NO}_{1100 \mathrm{~m} / \mathrm{min}}^{3}$ & $643+/-50$ & 32,6 \\
\hline $\mathrm{NOR}_{1100 \mathrm{~m} / \mathrm{min}}$ & $562,884+/-209,73$ & 30,0 \\
\hline $\mathrm{NO}_{1217 \mathrm{~m} / \mathrm{min}}$ & $747,04+/-116,81$ & 42,5 \\
\hline $\mathrm{NOR}_{1217 \mathrm{~m} / \mathrm{min}}$ & $525,8+/-90,44$ & 40,7 \\
\hline $\mathrm{NO}_{1480 \mathrm{~m} / \mathrm{min}}$ & $772+/-98$ & 35,8 \\
\hline $\mathrm{NOR}_{1480 \mathrm{~m} / \mathrm{min}}$ & $580+/-90$ & 34,2 \\
\hline
\end{tabular}

${ }^{1} \mathrm{NA}=$ nanofibras aleatorias.

$2 \mathrm{NAR}=$ nanofibras aleatorias recocidas.

${ }^{3} \mathrm{NO}=$ nanofibras ordenadas.

${ }^{4} \mathrm{NOR}=$ nanofibras ordenadas recocidas.

Las nanofibras de PLA presentan una diferencia en los diámetros de las nanofibras colectadas ordenada y aleatoriamente, siendo estas últimas las de mayor diámetro promedio $(992 \mathrm{~nm})$ con una variación de $26 \mathrm{~nm}$. Luego del recocido, estas nanofibras presentan un diámetro promedio de $986 \mathrm{~nm}$ con una variación de $21 \mathrm{~nm}$, lo cual no indica un cambio significante en este parámetro. Sin embargo, se presenta un aumento del $7 \%$ en la porosidad de la membrana, la cual es atribuida al aumento de los espacios vacíos dada la disminución del diámetro de las nanofibras. En el caso de las nanofibras ordenadas colectadas a $1100 \mathrm{~m} / \mathrm{min}\left(\mathrm{NO}_{1100 \mathrm{~m} / \mathrm{min}}\right)$ presentan un diámetro promedio de $643 \mathrm{~nm}$, el cual es $350 \mathrm{~nm}$ menor que las obtenidas con el colector estacionario, luego del recocido estas fibras disminuyen $80 \mathrm{~nm}$ su diámetro promedio, así como disminuyen en un $2 \%$ la porosidad de la membrana. Esta disminución puede ser otorgada a la gran aglomeración de nanofibras en la membrana luego del tratamiento térmico, lo cual genera una disminución de las zonas oscuras en la imagen, parámetro por el cual se mide la porosidad de la membrana.

Con relación a las nanofibras colectadas a 1217 $\mathrm{m} / \mathrm{min}\left(\mathrm{NO}_{1217} \mathrm{~m} / \mathrm{min}\right)$ se encuentra una disminución de $250 \mathrm{~nm}$ en el diámetro promedio en comparación con las nanofibras colectadas aleatoriamente y un aumento de $100 \mathrm{~nm}$ respecto a las nanofibras 
colectadas a $1100 \mathrm{~m} / \mathrm{min}$. Este incremento es otorgado a los parámetros de electrohilado como el potencial eléctrico y velocidad tangencial, los cuales al tener un mayor valor $(15 \mathrm{Kv}$ y $1217 \mathrm{~m} / \mathrm{min})$ tienen una gran influencia en el estiramiento del chorro expulsado por lo que el diámetro de las nanofibras aumenta. Al recocer las nanofibras de este tratamiento, el diámetro promedio y la porosidad de la membrana disminuye $220 \mathrm{~nm}$ y $2 \%$ respectivamente, siendo esto el resultado esperado dados los resultados obtenidos con las nanofibras colectadas a $1100 \mathrm{~m} / \mathrm{min}$.

Por último, el diámetro de las nanofibras colectadas a $1480 \mathrm{~m} / \mathrm{min}\left(\mathrm{NO}_{1480 \mathrm{~m} / \mathrm{min}}\right)$ presenta una disminución en el diámetro promedio de $220 \mathrm{~nm}$ con relación a las nanofibras colectadas aleatoriamente y un aumento en esta variable de 130 y $30 \mathrm{~nm}$ en comparación con las nanofibras colectadas a $1100 \mathrm{~m} / \mathrm{min}$ y $1217 \mathrm{~m} / \mathrm{min}$ respectivamente. Luego de realizar el tratamiento térmico a las fibras obtenidas a $1480 \mathrm{~m} /$ min, se presenta una disminución en el diámetro promedio de $200 \mathrm{~nm}$ aproximadamente, así como una leve disminución $(1,6 \%)$ en la porosidad de la membrana, siendo esto similar a lo observado en las fibras obtenidas a menor potencial eléctrico y menor velocidad tangencial.

De acuerdo a estos resultados es posible notar un incremento en el diámetro de las fibras colectadas ordenadamente a medida que la velocidad tangencial y el potencial eléctrico aumentan, además no se observa una tendencia con relación a la porosidad de las membranas, dado que en los tres tratamientos de las fibras colectadas ordenadamente la membrana que presenta la mayor porosidad $(42,5 \%)$ fue obtenida a una velocidad tangencial de $1217 \mathrm{~m} / \mathrm{min}$. También es posible notar una diferencia entre los diámetros de las nanofibras colectadas ordenadamente en comparación con las colectadas aleatoriamente, siendo estas últimas las que presentaron el mayor diámetro promedio. La disminución en el diámetro es otorgada al colector rotatorio, el cual, de acuerdo a la velocidad de colección, se genera un mayor o menor estiramiento de la fibra durante el proceso de electrohilado. Sin embargo, en los resultados se observó un aumento en el diámetro de las fibras a medida que aumentaba la velocidad tangencial, por lo tanto, se puede indicar que el voltaje es el factor determinante a la hora de obtener fibras con diámetros nanométricos.
Respecto al tratamiento térmico de las nanofibras, se observa una baja variación en el diámetro de las nanofibras colectadas aleatoriamente (NA), sin

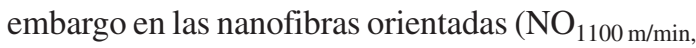
$\mathrm{NO}_{1217} \mathrm{~m} / \mathrm{min}, \mathrm{NO}_{1480 \mathrm{~m} / \mathrm{min}}$ ) se presenta una disminución en el diámetro promedio del $12,5 \%$, $29,6 \%$ y $24,87 \%$ respectivamente (Figura 3 ), la cual es otorgada al auto ensamble de las cadenas moleculares de PLA dispuestas en la nanofibra, dado que el tiempo y la temperatura aplicados permiten que las cadenas se organicen mejor para formar cristales más regulares.

La Figura 3 muestra el valor medio y la variación en los diámetros de las fibras obtenidas por los distintos tratamientos descritos anteriormente.

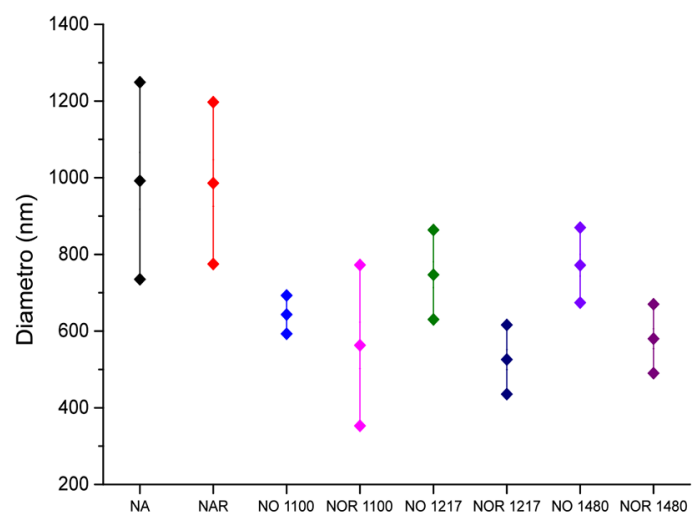

Figura 3. Efectos de la velocidad de colección y recocido en el diámetro de las fibras de PLA.

Se puede evidenciar una disminución en los máximos y mínimos de las fibras de casi todos los tratamientos. El tratamiento que está exento a esto es el tratamiento NOR 1100 dado que luego del recocido la desviación estándar de las fibras se ve incrementado, fenómeno que puede ser ocasionado por algunas fusiones de las fibras dado el tratamiento térmico. Con esto es posible indicar que el recocido es parte importante a la hora de obtener fibras con menor diámetro.

\section{Morfología por microscopia electrónica de transmisión}

En la Figura 4 se presentan las imágenes TEM obtenidas de la sección transversal de las nanofibras 
colectadas a $1100 \mathrm{~m} / \mathrm{min}$ y $1480 \mathrm{~m} / \mathrm{min}$. En las imágenes obtenidas es posible notar cambios y diferencias en la parte interna de las nanofibras, las cuales dadas las imágenes presentan un núcleo y una corteza.

En la Figura 4A se pueden observar nanofibras con diámetros homogéneos y de una sola tonalidad, mientras que en la Figura 3C, se observa con mayor nitidez el núcleo de las nanofibras NO $1100 \mathrm{~m} / \mathrm{min}$ de una tonalidad oscura y la parte externa más clara.

A partir de lo observado es posible tener un indicio de la conformación y estructura interna de las nanofibras, en donde se asume que el núcleo presenta una fase con características amorfas mientras la corteza o parte externa de las nanofibras presentaría un estado cristalino. Además, dadas las imágenes se pueden notar dos tipos de nanofibras, las cuales son nanofibras que presentan diámetros grandes con una estructura distinguible y otras pocas con diámetros pequeños con una corteza delgada. Posiblemente esto sea un reflejo de que no todas las nanofibras tienen las mismas condiciones de electrohilado, debido a que algunas al momento de adherirse al tambor llegan más rápido que otras, lo que les impide estirarse y adelgazar [10]. Otra posible causa puede ser otorgada al movimiento de las nanofibras las cuales son afectadas por el movimiento turbulento del aire producido por la rotación del colector, generando una diferencia en el proceso de curado para cada una de ellas.

En cuanto a las nanofibras con recocido (Figura 5), se observan las nanofibras recocidas obtenidas con las tres velocidades de colección utilizadas en el electrohilado. En las imágenes se notan mayoritariamente fibras con un núcleo bastante homogéneo, lo que indica que se presentaron las condiciones de temperatura y tiempo adecuadas

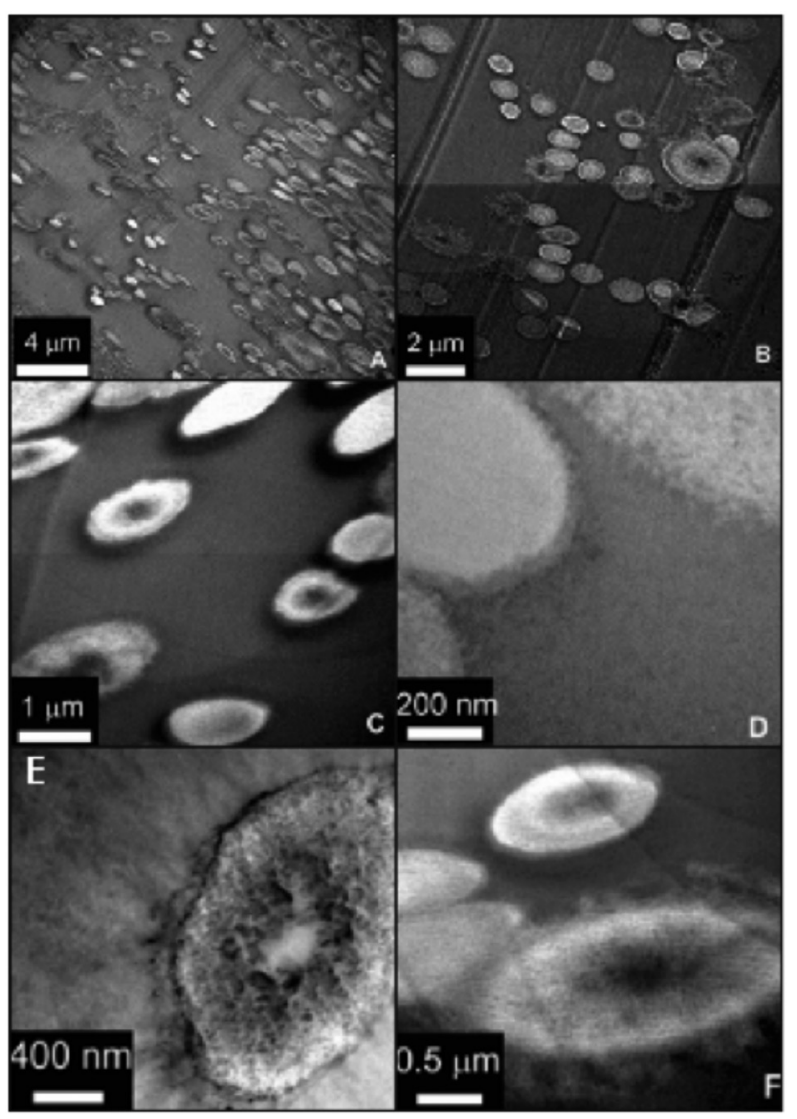

Figura 4. Microscopia electrónica de transmisión de nanofibras orientadas a $1100 \mathrm{~m} / \mathrm{min}$ (A, C y E) y nanofibras orientadas a $1480 \mathrm{~m} / \mathrm{min}(\mathrm{B}, \mathrm{D}, \mathrm{y} \mathrm{F})$. 


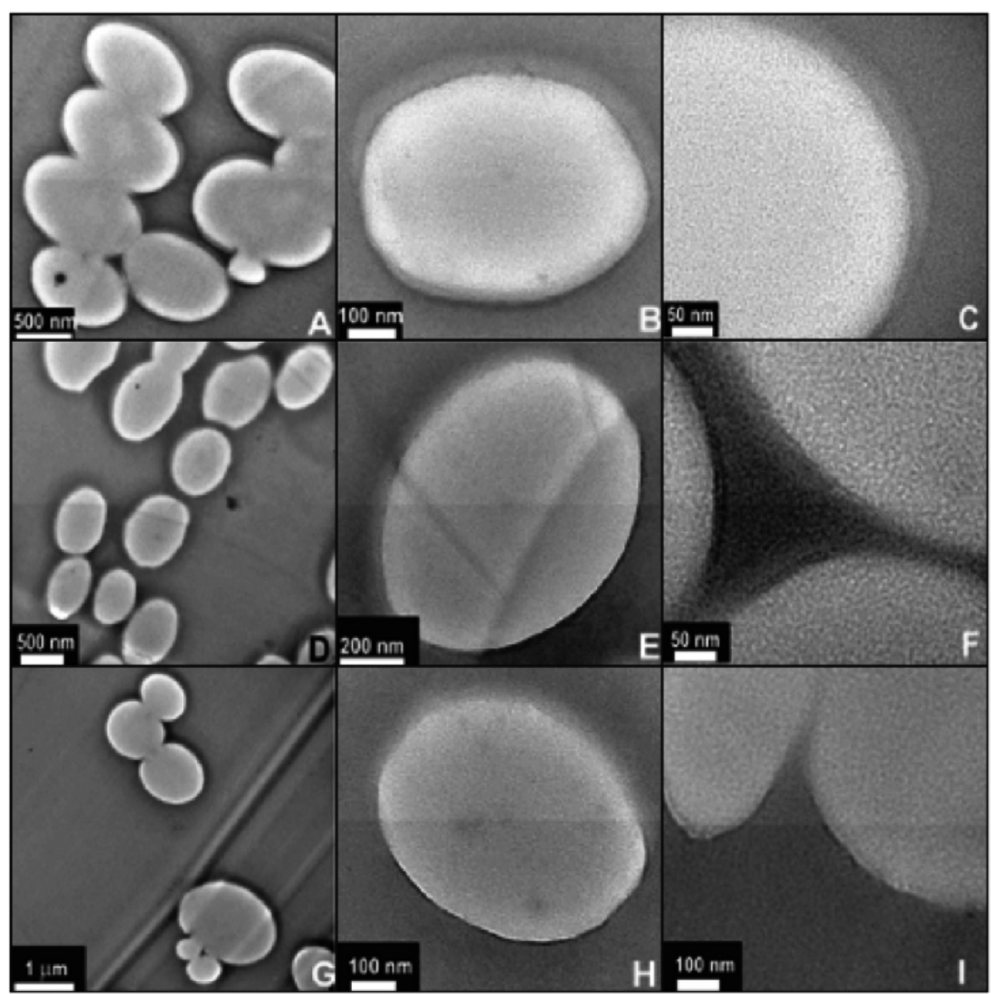

Figura 5. Observación de la estructura de las nanofibras orientadas recocidas mediante micrografías de TEM a diferentes aumentos. A), B) y C) sección transversal de NO $1100 \mathrm{~m} / \mathrm{min}$. D), E) y F) sección transversal de NO $1217 \mathrm{~m} / \mathrm{min}$. G), H) y I) sección transversal de NO $1480 \mathrm{~m} / \mathrm{min}$.

para reorganizar las cadenas poliméricas, mejorando el grado de cristalinidad del polímero. Esto puede generar un mejoramiento en las propiedades mecánicas del material. La mencionada delgada capa de corteza, se puede observar claramente en la Figura 4 (C, F).

\section{Estructura cristalina}

En los resultados de DRX obtenidos por Gómez et al. [16], se presenta evidencia de un incremento en el estado amorfo de las nanofibras al ser colectadas ordenadamente, sin esta mayor a una mayor velocidad tangencial, pero luego del recocido de las nanofibras se presenta una señal definida a los $16,8^{\circ}$, por lo tanto es evidente que el tratamiento térmico realizado promueve una cristalización extensiva de las cadenas poliméricas. Estos resultados también presentan una gran similitud a lo observado en el difractograma de PLA grado ACS, además de ser similares a los reportados por Inai et al. [13].
Para obtener un análisis detallado sobre la estructura de las nanofibras, fue necesario realizar técnicas que presenten y revelen detalles a nivel nanométrico, por ello, mediante el análisis SAXS realizado a las nanofibras es posible determinar la conformación o estructura cristalina del polímero. La Figura 6 presenta los resultados obtenidos luego del análisis SAXS realizado tanto al PLA comercial como a las nanofibras colectadas aleatoria y ordenadamente $(1480 \mathrm{~m} / \mathrm{min})$.

$\mathrm{Al}$ igual que los resultados de DRX, los espectros obtenidos por SAXS de los andamios de nanofibras aleatorias (NA) y de nanofibras orientadas $\left(\mathrm{NO}_{1480 \mathrm{~m} / \mathrm{min}}\right)$ presentan una diferencia significativa con relación al espectro de PLA grado ACS. Al enfocar la atención en las nanofibras, se observa un corrimiento de la señal correspondiente al tamaño de cristal de las nanofibras colectadas ordenadamente. 


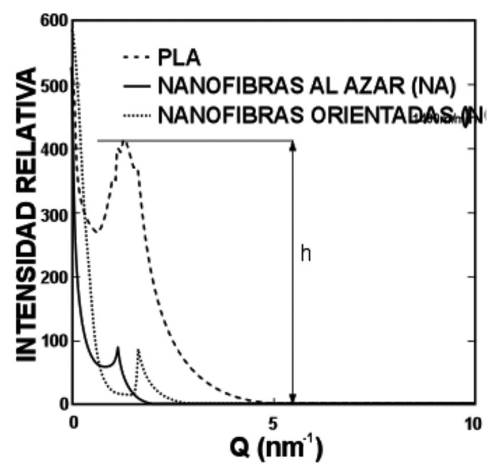

Figura 6. Espectros del PLA grado ACS, de las nanofibras colectadas aleatoriamente y de las nanofibras orientadas a una velocidad de $1480 \mathrm{~m} / \mathrm{min}$.

A partir de la curva de la Figura 7 se obtiene la distancia entre bloques cristalinos y el tamaño de los cristales del polímero en el material [17]. Se grafican las intensidades relativas en función del vector de dispersión $(\mathrm{Q})$. A partir de la ecuación de dispersión (1), se calcula el valor del ángulo $(\theta)$ donde se generó la difracción, obteniendo Q de la figura y con una longitud de onda de rayos $\mathrm{X}$ incidente de $\lambda=1.5418 \AA$.

$$
Q=\frac{4 \pi}{\lambda} \operatorname{seno}\left(\frac{\theta}{2}\right)
$$

Se calculó la distancia entre bloques a partir de la ecuación (2).

$$
d=\frac{\lambda}{2 \operatorname{seno}\left(\frac{\theta}{2}\right)}
$$

Para el cálculo del tamaño del cristal, se usa la ecuación (4).

$$
\begin{gathered}
B=\frac{K \lambda}{S \cos (\theta)} \\
S=\frac{K \lambda}{B \cos (\theta)}
\end{gathered}
$$

Donde K es considerado como una constante igual a $0,94, S$ es el tamaño del cristal y B es el ancho de la línea a media altura máxima.
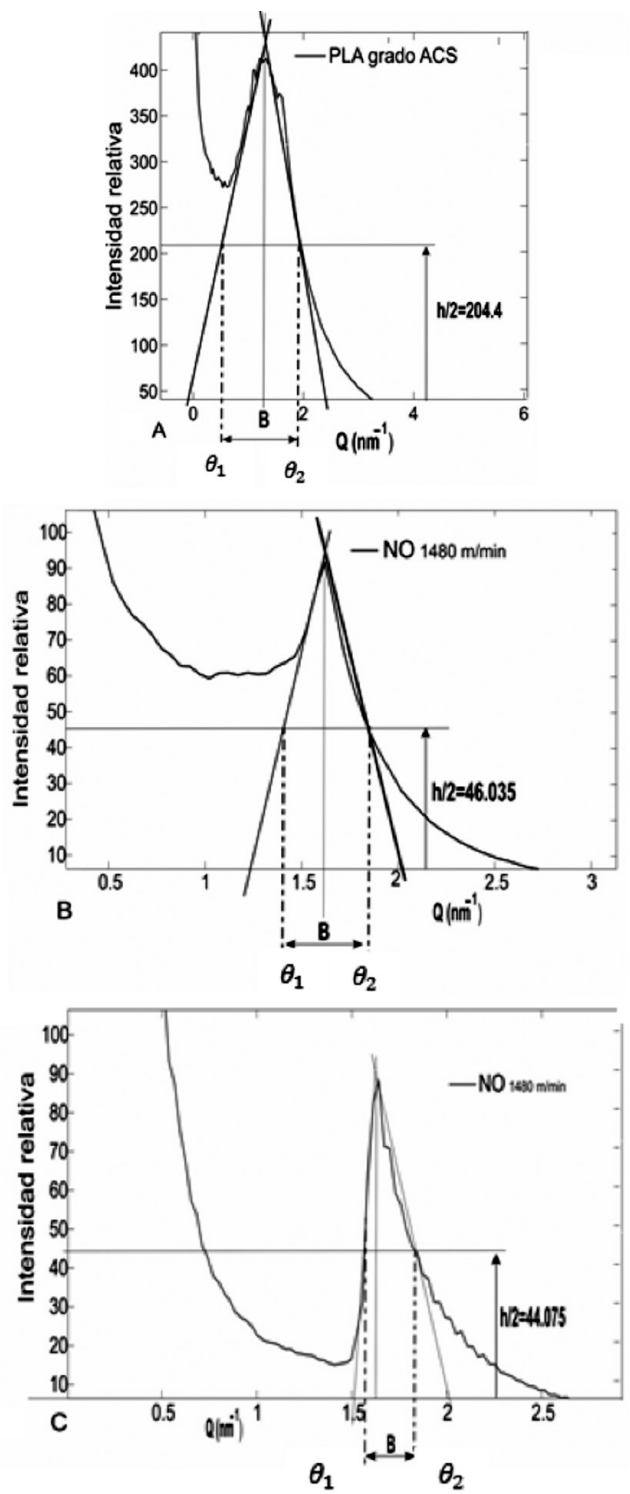

Figura 7. Geometrías para el cálculo de B y el cálculo del tamaño del cristal A.

Como en el caso actual los picos de dispersión no son simétricos, se debe proyectar líneas tangentes a las curvas y su corte con $\mathrm{Q}$, determinan $\theta_{1}$ y $\theta_{2}$, donde $B=\theta_{2}-\theta_{1}$ (expresado en radianes). En la Figura 10, se observan las líneas que resumen este procedimiento, aplicado a las 3 dispersiones.

En la Tabla 4 se resumen los valores para cada material probado por SAXS. Los resultados indican que el proceso de electrohilado aumenta hasta 4 veces el tamaño de los cristales, desde el PLA en 
Tabla 4. Valores cristalográficos de las nanofibras obtenidas por SAXS.

\begin{tabular}{|l|c|c|c|}
\hline \multirow{2}{*}{ Parámetro } & \multicolumn{2}{c|}{ PLA } \\
\cline { 2 - 4 } & Pellets & Nanofibras aleatorias & Nanofibras ordenadas a 1480m/min \\
\hline $\mathrm{Q}\left(\mathrm{NM}^{-1}\right)$ & 1,264 & 1,620 & 1,642 \\
\hline Angulo $(\Theta)$ & 0,0310 & 0,0397 & 0,0403 \\
\hline Distancia entre bloques cristalinos $(\mathrm{d})$ & 4,971 & 3,879 & 3,827 \\
\hline $\mathrm{B}=\left(\Theta_{1}-\Theta_{2}\right) \operatorname{Rad}$ & 0,00124 & $3,167 \mathrm{e}^{-4}$ & $2,654 \mathrm{e}^{-4}$ \\
\hline Tamaño de cristal $(\mathrm{s}) \mathrm{nm}$ & 11,66 & 45,74 & 54,59 \\
\hline
\end{tabular}

grado ASC hasta las nanofibras orientadas. Por otro lado, la nanofibra aumenta 5 veces el tamaño del cristal, cuando está orientada. Por esta razón no es detectable en DRX, siendo tan grande el tamaño de estos cristales no se pueden difractar los rayos $\mathrm{X}$ en las nanofibras. Por otro lado, se observa que el electrohilado disminuye la distancia entre bloques cristalinos y al orientar la nanofibra se disminuye más. La presencia de cristales grandes con menos distancia entre ellos indica una orientación de las nanofibras en su estructura interna.

\section{Transiciones térmicas}

En los análisis de DSC presentados por Gómez et al. [16] (Figura 8) se muestra una variación entre el tipo de colección y la velocidad de colección. Los pellets de PLA presentan un $\mathrm{Tg}$ a $\operatorname{los} 60^{\circ} \mathrm{C}$, una cristalización cercana a $100^{\circ} \mathrm{C}$ y luego una última señal a $150^{\circ} \mathrm{C}$. Este mismo patrón lo sigue las nanofibras colectadas aleatoriamente, sin embargo el último punto de fusión de las fibras con y sin recocido presenta dos señales, las cuales son otorgadas a la fusión de los componentes $\alpha$ y $\alpha^{\prime}[18]$, por lo tanto se puede indicar que este método de colección genera fibras con PLA de dos diferentes estructuras. En esta temperatura se presenta inicialmente la fusión de la forma $\alpha^{\prime}$ y su recristalización en la forma $\alpha$, mientras que la segunda señal corresponde a la fusión de la forma $\alpha$ [19].

Allí también es posible dilucidar el grado de desorden molecular de las cadenas el cual es reflejado por la relajación entálpica, siendo esta alta en las NA en comparación con NAR y PLA grado ACS, que muestran valores de entalpia de $6,3 \mathrm{~J} / \mathrm{g}, 5,8 \mathrm{~J} / \mathrm{g}$ y $2,4 \mathrm{~J} / \mathrm{g}$, respectivamente.

Con relación a los termogramas de las nanofibras colectadas ordenadamente NO (Figura 8B) también se muestran picos de relajación entálpica alrededor de $\operatorname{los} 65^{\circ} \mathrm{C}$, con una entalpía de $5,8 \mathrm{~J} / \mathrm{g}$ para $\mathrm{NO}_{1100 \mathrm{~m} / \mathrm{min}}, \mathrm{NO}_{1217 \mathrm{~m} / \mathrm{min}}$ y $\mathrm{NO}_{1480 \mathrm{~m} / \mathrm{min}}$, valor que presenta similitud al observado en las nanofibras colectadas aleatoriamente. En el termograma de las nanofibras ordenadas (NO) no se observa temperatura de cristalización como el encontrado en la Figura 8A y una sola señal en su temperatura de
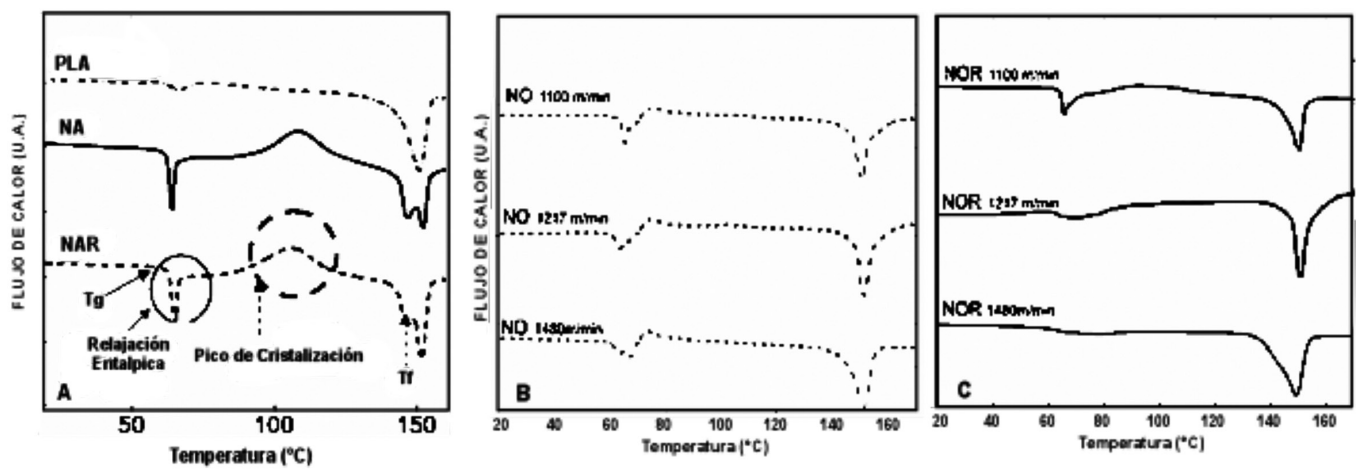

Figura 8. Calorimetría diferencial de barrido (A) PLA grado ACS y nanofibras colectadas aleatoriamente sin y con tratamiento térmico respectivamente (NA) y (NAR), (B) Nanofibras de PLA orientadas ( $\mathrm{NO}_{1100 \mathrm{~m} / \mathrm{min},} \mathrm{NO}_{1217 \mathrm{~m} / \mathrm{min},} \mathrm{NO}_{1480 \mathrm{~m} / \mathrm{min}}$ ) y (C) Nanofibras de PLA orientadas con recocido $\left(\mathrm{NOR}_{1100 \mathrm{~m} / \mathrm{min},} \mathrm{NOR}_{1217 \mathrm{~m} / \mathrm{min} \text { y }} \mathrm{NOR}_{1480 \mathrm{~m} / \mathrm{min}}\right)$. 
fusión, por lo cual se puede indicar que este proceso de colección provoca una sola estructuración del polímero, la cual viene siendo la estructura $\alpha$ dada la temperatura requerida para la fusión.

Las nanofibras ordenadas y recocidas (NOR) presentan una diferencia con la Figura 8B, con relación a la temperatura de transición vítrea, dado se observa una tendencia la cual disminuye la energía de transición vítrea a medida que se incrementa la velocidad de colección, llegando hasta el punto de desaparecer la Tg, tal y como se observa con el termograma de las nanofibras recocidas colectadas a $1480 \mathrm{~m} / \mathrm{min}$.

En la Tabla 5 se resumen los valores de la entalpia de cristalización, de fusión y el cálculo del porcentaje de cristalinidad tanto del PLA comercial en pellets, como en fibras obtenidas aleatorias y ordenadas.

\section{Propiedades mecánicas de los andamios de PLA por tracción uniaxial}

En las Figura 9A y 9B, se observa que las nanofibras colectadas aleatoriamente se presenta un pico bastante pronunciado después del punto de "fluencia" de la red en comparación con las nanofibras alineadas. Este punto llamado de "fluencia" refleja la rotura masiva de las nanofibras que se alinearon en la dirección de la carga para ambos tipos de andamios. Sin embargo, las nanofibras dispuestas aleatoriamente presentan una menor probabilidad de tener nanofibras alineadas en el andamio inicial y por lo tanto son muy pocas las fibras que se alinean para soportar la carga, mientras que en el caso de nanofibras orientadas (NO) existe una gran cantidad de fibras en la misma dirección generando una mayor resistencia a la rotura.
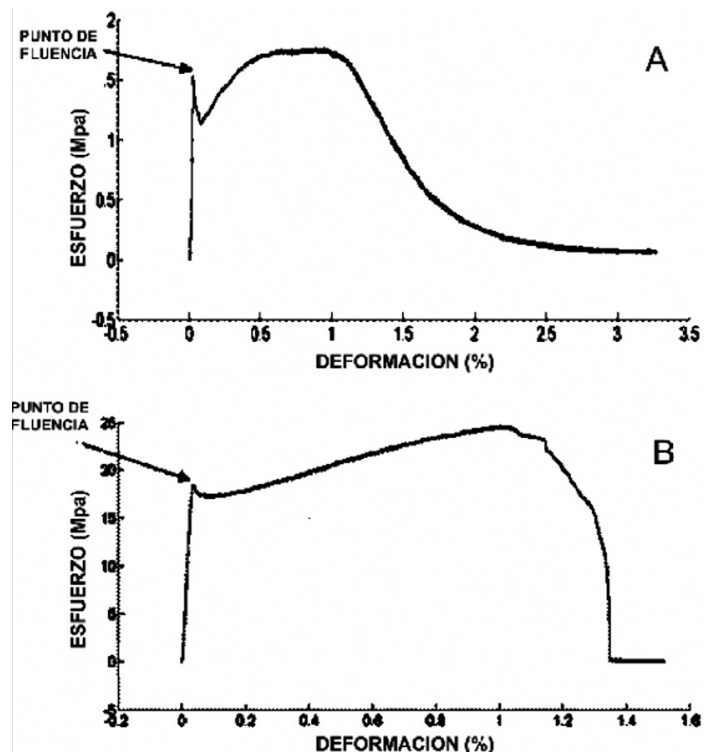

Figura 9. Comportamiento mecánico (A) nanofibras dispuestas al azar y (B) nanofibras orientadas $\mathrm{NO}_{1100 \mathrm{~m} / \mathrm{min}}$.

En la Tabla 6, se presentan los resultados de los ensayos de tracción a los diferentes andamios. Los datos del módulo elástico presentan variabilidad debido a la estructura del andamio influenciada por distribución de poros, densidad, distribución

Tabla 5. Grado de cristalinidad de las nanofibras electrohiladas.

\begin{tabular}{|l|c|c|c|c|}
\hline \multicolumn{1}{|c|}{ Tipo de PLA } & $\Delta H_{f}$ & $\Delta H_{c}$ & $\begin{array}{c}\text { Cristalinidad considerando } \\
\text { la cristalización } \\
=\frac{\chi_{b c}}{\Delta H_{f}}\end{array}$ & $\begin{array}{c}\text { Cristalinidad después } \\
\text { de la cristalización }\end{array}$ \\
\hline PLA grado ACS & 28,60 & N.E & 30,75 & $\chi_{a c}=\frac{\Delta H_{f}}{\Delta H_{f}^{0}}$ \\
\hline NA & 21,20 & 19,43 & 1,90 & 30,75 \\
\hline NAR & 25,67 & 23,13 & 2,73 & 22,8 \\
\hline $\mathrm{NO}_{1100} \mathrm{~m} / \min$ & 28,63 & 3,947 & 26,54 & 27,60 \\
\hline $\mathrm{NOR}_{1100 \mathrm{~m} / \min }$ & 19,39 & 7,69 & 12,58 & 30,78 \\
\hline $\mathrm{NO}_{1217} \mathrm{~m} / \mathrm{min}$ & 31,93 & 4,604 & 29,38 & 20,9 \\
\hline $\mathrm{NOR}_{1217} \mathrm{~m} / \mathrm{min}$ & 29,84 & $\mathrm{~N} . \mathrm{E}$ & 32,09 & 29,38 \\
\hline $\mathrm{NO}_{1480 \mathrm{~m} / \mathrm{min}}$ & 29,43 & 4,109 & 27,22 & 32,09 \\
\hline $\mathrm{NOR}_{1480 \mathrm{~m} / \mathrm{min}}$ & 29,89 & $\mathrm{~N} . \mathrm{E}$ & 32,14 & 31,64 \\
\hline
\end{tabular}


de nanofibras y presencia de cúmulos con diferentes tamaños.

En la Figura 10, se realiza la comparación de los resultados de tracción. El módulo elástico y el esfuerzo a la tracción de los andamios con nanofibras orientadas (NO) fueron 7-9 veces y $10-13$ veces respectivamente, mayor que los andamios con nanofibras dispuestas al azar (NA). Estas diferencias en las propiedades elásticas se explican en términos de la orientación molecular y de las nanofibras. En contraste, a los andamios con nanofibra aleatoria (NA), las nanofibras orientadas (NO) están orientadas paralelas a la dirección de carga. Por lo tanto, casi todas las nanofibras soportan y distribuyen la carga de forma simultánea durante el ensayo de tracción uniaxial.

Además, la orientación de las cadenas poliméricas sugerida por las pruebas de caracterización es mayor en las (NO) que en las (NA) debido al estiramiento de las (NO) durante el proceso de electrohilado. Como las cadenas de los polímeros se orientan a lo largo del eje principal de las nanofibras, el mejoramiento del módulo elástico en la dirección de las nanofibras es debido a la presencia de los fuertes enlaces covalentes entre los átomos de carbono en la cadena de polímero, en comparación con los enlaces intermoleculares entre las cadenas del polímero del PLA (enlaces de Van der Waals, enlaces de hidrógeno y otros). Durante el curso de los ensayos de tensión, las nanofibras de las NA en andamios fueron orientadas a lo largo de la dirección de la carga

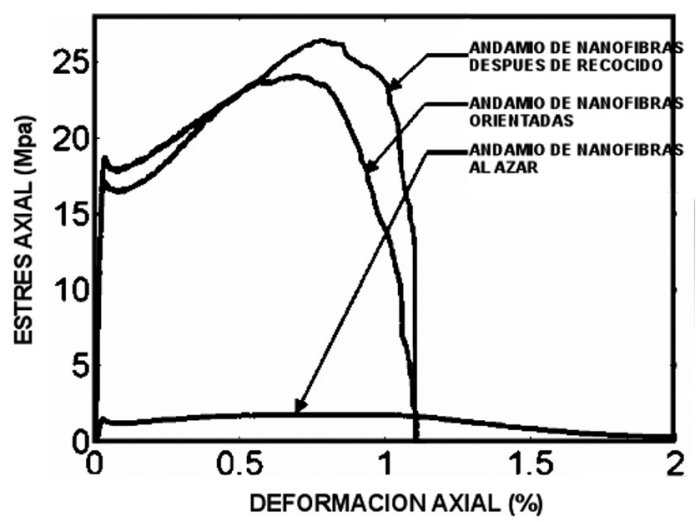

Figura 10. Ensayos de tracción de andamios con (NA) y andamios con $\left(\mathrm{NO}_{1100 \mathrm{~m} / \mathrm{min}}\right)$. aplicada y por ello algunas nanofibras fallaron mientras otras soportaron la carga aplicada hasta que se produce la fractura final. Como resultado, los andamios con NA presentan los más pequeños esfuerzos de limite elástico $\sigma_{\mathbf{y s}} \mathrm{y}$ resistencia a la tensión $\sigma_{\text {us }}$ (esfuerzo último), en comparación con los andamios de nanofibras orientadas (NO).

El módulo elástico de Young de las (NO) colectado a $1480 \mathrm{~m} / \mathrm{min}$, es más alto que las colectadas a $1217 \mathrm{~m} / \mathrm{min}$ y que las colectadas a $1100 \mathrm{~m} / \mathrm{min}$, esto indica que a mayor rotación se genera una disminución del tamaño de cristal lo que influirá en el aumento del módulo de Young. En la Figura 11 se observa que las nanofibras con recocido $\mathrm{NOR}_{1217 \mathrm{~m} / \mathrm{min}}$ no presentaron un efecto en las propiedades elásticas de las nanofibras orientadas $\left(\mathrm{NO}_{1217 \mathrm{~m} / \mathrm{min}}\right)$, ya que la alta desviación estándar en los resultados de ambos tipos de nanofibras muestra que son estadísticamente iguales (881.3+/-100.2 y 854.2+ /-60.7 para $\left.\mathrm{NO}_{1217} \mathrm{~m} / \mathrm{min} \mathrm{y} \mathrm{NO}_{1217 \mathrm{~m} / \mathrm{min}}\right)$. Este hecho sugiere que el cambio en la cristalización no afecta las propiedades elásticas de las nanofibras orientadas.

Esto se puede explicar porque el recocido genera una homogenización de los cristales hacia todas direcciones, por lo que no generaría un mejoramiento preferencial de la orientación de los cristales respecto de las nanofibras orientadas sin recocidas en la dirección de la aplicación

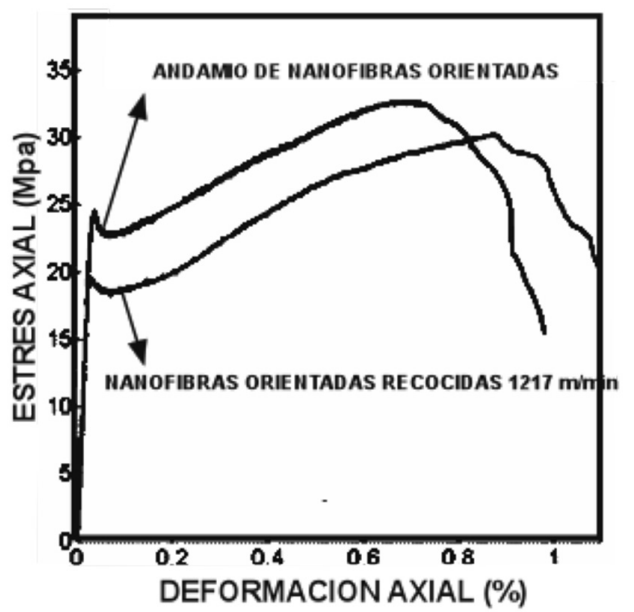

Figura 11. Comparación de resultados de los ensayos de tracción de andamios con $\mathrm{NO}_{1217} \mathrm{~m} / \mathrm{min}$. 
Tabla 6. Resultados de los ensayos de tracción en los diferentes tipos de nanofibras.

\begin{tabular}{|l|c|c|l|c|c|}
\hline \multicolumn{1}{|c|}{$\begin{array}{c}\text { Tipo de } \\
\text { Andamio }\end{array}$} & $\begin{array}{c}\text { Módulo de } \\
\text { Young } \\
\text { E (MPa) }\end{array}$ & $\begin{array}{c}\text { Esfuerzo de } \\
\text { fluencia } \sigma_{\mathbf{y s}} \\
(\mathbf{M P a})\end{array}$ & $\begin{array}{c}\text { Deformación a } \\
\text { la fluencia \% }\end{array}$ & $\begin{array}{c}\text { Resistencia a } \\
\text { la tensión } \sigma_{\text {us }} \\
(\mathbf{M P a})\end{array}$ & $\begin{array}{c}\text { Deformación } \\
\text { ultima } \\
\text { max \% }\end{array}$ \\
\hline $\mathrm{NA}$ & $96,8+/-32,5$ & $1,7+/-0,2$ & $4,2+/-2,4$ & $2,8+/-0,6$ & $129,6+/-32,5$ \\
\hline $\mathrm{NO}_{1100 \mathrm{~m} / \mathrm{min}}$ & $683,6+/-33,2$ & $18,4+/-0,6$ & $3,4+/-0,3$ & $24,0+/-0,7$ & $86,4+/-15,2$ \\
\hline $\mathrm{NOR}_{1100 \mathrm{~m} / \mathrm{min}}$ & $620,8+/-74,2$ & $13,8+/-4,8$ & $3,6+/-0,4$ & $20,3+/-8,8$ & $95,5+/-10,0$ \\
\hline $\mathrm{NO}_{1217} \mathrm{~m} / \mathrm{min}$ & $881,3+/-100,2$ & $25,0+/-2,2$ & $3,7+/-0,4$ & $32,2 /-2,9$ & $75,0+/-7$ \\
\hline $\mathrm{NOR}_{1217 \mathrm{~m} / \mathrm{min}}$ & $854,2+/-60,7$ & $19,4+/-1,1$ & $3,5+/-0,7$ & $31,0+/-3,0$ & $93,0+/-4,9$ \\
\hline $\mathrm{NO}_{1480} \mathrm{~m} / \mathrm{min}$ & $1026,73+/-320$ & $29,23+/-2,2$ & $4,73+/-1,7$ & $30,29+/-2,5$ & $75,2+/-1,9$ \\
\hline $\mathrm{NOR}_{1480} \mathrm{~m} / \mathrm{min}$ & $1177,4+/-106$ & $31,68+/-4,25$ & $3,549+/-0,69$ & $44,43+/-12,5$ & $80,17+/-39,5$ \\
\hline
\end{tabular}

de la carga. Estos resultados contrastan con los obtenidos por C.T Lim et al. [20], donde indicaron un mejoramiento de las propiedades elásticas de nanofibras orientadas al azar de Policaprolactona por efecto del tratamiento térmico, pero en ese estudio no se compara con nanofibras orientadas, y como se ha indicado en el presente estudio la orientación de nanofibras genera una orientación molecular que afectarán el módulo de Young.

\section{CONCLUSIONES}

Mediante la aplicación de diferentes técnicas de caracterización se logró determinar las propiedades morfológicas y estructurales de las nanofibras con lo cual fue posible obtener una descripción completa y detallada de la estructura de las nanofibras de PLA y su relación con variables tales como la orientación de nanofibras en los andamios, velocidad de rotación del colector y tratamientos térmicos posteriores al electrohilado. Mediante esta experimentación se evidencio la relación y los cambios que genera el tipo de colector, siendo mejor el colector rotatorio debido a que disminuye los defectos en las fibras. En cuanto al recocido se presenta una mejora en la cristalinidad, sin embargo, no existe cambio considerable en las propiedades elásticas como en la respuesta a la resistencia a tensión. Este trabajo permitió observar las propiedades elásticas de nanofibras, a partir de las variables mencionadas, lo que se puede usar a futuro en el diseño de los andamios celulares de nanofibras de PLA. Además, las condiciones en que se realizó el electrohilado son aceptables y muy cercanos a los ideales.

\section{AGRADECIMIENTOS}

Se agradece el apoyo del Instituto de Investigaciones en Materiales, UNAM, así como de sus técnicos académicos por el apoyo en la realización de los análisis: Adriana Tejeda (DRX), Eriseth Fregoso (DSC), Omar Novelo and Esau González (SEM), Carlos Flores (TEM). Yiguel Ángel Canseco (ATRFTIR). Se agradece también a DGAPA, UNAM por el apoyo económico a este trabajo a través del proyecto PAPIIT IN 108116.

\section{REFERENCIAS}

[1] C.P. Barnes, S.A. Sell, E.D. Boland, D.G. Simpson, and G.L. Bowlin. "Nanofiber technology: Designing the next generation of tissue engineering scaffolds". Advanced Drug Delivery Reviews. Vol. 59, pp. 14131433. 2007.

[2] G. Chen, T. Ushida, and T. Tateishi. "Scaffold Design for Tissue Engineering". Macromolecular Bioscience. Vol. 2, pp. 67-77. 2002.

[3] B.D. Ulery, L.S. Nair, and C.T. Laurencin. "Biomedical applications of biodegradable polymers". Journal of Polymer Science Part B: Polymer Physics. Vol. 49, pp. 832-864. 2011.

[4] N. Ninan, M. Muthiah, I.-K. Park, A. Elain, T. W. Wong, S. Thomas, et al. "Faujasites incorporated tissue engineering scaffolds for wound healing: in vitro and in vivo analysis". ACS applied materials \& interfaces. Vol. 5, pp. 11194-11206. 2013.

[5] L. Kong, Y. Gao, G. Lu, Y. Gong, N. Zhao, and X. Zhang. "A study on the bioactivity 
of chitosan/nano-hydroxyapatite composite scaffolds for bone tissue engineering". European Polymer Journal. Vol. 42, pp. 3171-3179. 2006.

[6] F.P. La Mantia, R. Arrigo, and M. Morreale. "Effect of the orientation and rheological behaviour of biodegradable polymer nanocomposites". European Polymer Journal. Vol. 54, pp. 11-17. 2014.

[7] K. Ghosal, M.S. Latha, and S. Thomas. "Poly(ester amides) (PEAs) - Scaffold for tissue engineering applications". European Polymer Journal. Vol. 60, pp. 58-68. 2014. R. Vasita and D.S. Katti. "Nanofibers and their applications in tissue engineering". International Journal of Nanomedicine. Vol. 1, pp. 15-30. 2006.

[9] C.T. Laurencin, S.G. Kumbar, S.P. Nukavarapu, R. James, and M.V. Hogan. "Recent patents on electrospun biomedical nanostructures: an overview". Recent patents on biomedical engineering. Vol. 1, pp. 68-78. 2008.

[10] L.M. D. Sánchez, L. Rodriguez, and M. López. "Electrospinning: la era de las nanofibras". Revista Iberoamericana de Polímeros. Vol. 14, pp. 10-27. 2013.

[11] T. Stylianopoulos, C.A. Bashur, A.S. Goldstein, S.A. Guelcher, and V.H. Barocas. "Computational predictions of the tensile properties of electrospun fibre meshes: effect of fibre diameter and fibre orientation". Journal of the mechanical behavior of biomedical materials. Vol. 1, pp. 326-335. 2008.

[12] R. Vera-Graziano, A. Maciel-Cerda, E. Moreno-Rondon, A. Ospina, and E. GomezPachon. "Modified polylactide microfiber scaffolds for tissue engineering". In MRS Proceedings. 2012, pp. imrc11-1376-s11-09.
[13] R. Inai, M. Kotaki, and S. Ramakrishna. "Structure and properties of electrospun PLLA single nanofibres". Nanotechnology. Vol. 16, p. 208. 2005.

[14] E.P.S. Tan, S.Y.Ng, and C.T. Lim. "Tensile testing of a single ultrafine polymeric fiber". Biomaterials. Vol. 26, pp. 1453-1456. 2005.

[15] Y. You, S. Won Lee, S. Jin Lee, and W. H. Park. "Thermal interfiber bonding of electrospun poly(l-lactic acid) nanofibers". Materials Letters. Vol. 60, pp. 1331-1333. 2006/05/01/ 2006.

[16] E.Y. Gómez-Pachón, F.M. Sánchez-Arévalo, F.J. Sabina, A. Maciel-Cerda, R.M. Campos, N. Batina, et al. "Characterisation and modelling of the elastic properties of poly(lactic acid) nanofibre scaffolds". Journal of Materials Science. Vol. 48, pp. 8308-8319. 2013.

[17] R. Parmar, M. Mangrola, B. Parmar, and V. Joshi. "A software to calculate crystalline size by Debey-Scherrer Formula using VB.NET". Multi Disciplinary Edu Global Quest. Vol. 1, 2012.

[18] P. Pan, B. Zhu, W. Kai, T. Dong, and Y. Inoue. "Polymorphic Transition in Disordered Poly(1lactide) Crystals Induced by Annealing at Elevated Temperatures". Macromolecules. Vol. 41, pp. 4296-4304. 2008/06/01 2008.

[19] P. Pan, W. Kai, B. Zhu, T. Dong, and Y. Inoue. "Polymorphous Crystallization and Multiple Melting Behavior of Poly(1lactide): Molecular Weight Dependence". Macromolecules. Vol. 40, pp. 6898-6905. 2007/09/01 2007.

[20] C. Lim, E. Tan, and S. Ng. "Effects of crystalline morphology on the tensile properties of electrospun polymer nanofibers". Applied Physics Letters. Vol. 92, p. 141908. 2008. 